\title{
Evaluation of Acute Toxicity and Symptoms Palliation in a Hypofractionated Weekly Schedule of External Radiotherapy for Elderly Patients with Muscular Invasive Bladder Cancer
}

Vassilis Kouloulias, Maria Tolia, Nikolaos Kolliarakis, Argyris Siatelis, Nikolaos Kelekis

Radiotherapy Unit, 2nd Radiology Department (KV, TM, KN) and Urology Department (SA), Attikon Hospital, Xaidari and Radiotherapy Department, Andreas Syggros Hospital, (KN), Athens, Greece

\section{ABSTRACT}

Aim: To evaluate acute toxicity and symptoms palliation of a weekly hypofractionated 3DCRT schedule as radical treatment in elderly patients with organ confined bladder cancer cT1-2N0.

Materials and Methods: Between February 2005 and June 2011, 58 prospectively selected patients diagnosed with organ confined bladder cancer were treated with external 3DCRT (4-field arrangement). All candidates were medically inoperable, with poor performance status, and with age ranged from 75 to 88 years (median 78). A dose of 36 Gy in 6 weekly fractions was prescribed. The primary study endpoints were the evaluation of haematuria, dysuria, frequency and pain palliation as well as the acute toxicity according to the RTOG/EORTC scale: an assessment was performed at baseline, during and 3 months after radiotherapy, while the maximum reported score was taken into account.

Results: The gastrointestinal acute toxicities were 13/58 (22.4\%) and 5/58 (5.6\%), for grade I and II respectively. The genitourinary acute toxicities were 19/58 (32.7\%) and $10 / 58$ (17.2\%), for grade I and II respectively. In terms of clinical outcome, 55/58 patients $(94.8 \%)$ reported palliation of haematuria, while 19 out of 58 reported no change in frequency and dysuria. All patients reported significant improvement $(\mathrm{P}<0.01)$ for pain, concerning the visual analogue score before and after radiotherapy. The median progression free survival was 14 months.

Conclusions: The incidence of patient-reported acute toxicity following weekly hypofractionated external 3DCRT is low while the symptom palliation compares very favorably with other reported outcomes.

\section{ARTICLE INFO}

\section{Key words:}

Urinary Bladder Neoplasms; Frail Elderly; Patients;

Radiotherapy; Toxicity Tests, Acute; Palliative Care

Int Braz J Urol. 2013; 39: 77-82

Submitted for publication:

May 31, 2012

Accepted after revision:

December 20, 2012

\section{INTRODUCTION}

Bladder cancer represents the fourth most common cancer and is three times more common in men than in women in United States. It is rarely diagnosed in individuals younger than 40 years.
Because the median age of diagnosis is 65 years, medical comorbidities are a frequent consideration in patient management (1). Bladder cancer is estimated to have an annual incidence in the United States of 68,810 cases, accounting for 5\% of all newly diagnosed cancers. Approximately 
14,100 people per year will die of this disease, accounting for $2.5 \%$ of all cancer-related mortality in the United States and 3\% of all cancer deaths in men (2). The highest incidence rates are in Western countries (3). From the early 1970 s to 2004 the incidence of this cancer rose slightly while mortality fell. This phenomenon is likely attributable to the increased detection of localized lesions and improved therapies (4).

A group of patients encompasses the invasive lesions, and the goal is to determine if the bladder should be removed or preserved without compromising survival and to determine if the primary lesion can be managed independently or if patients are at high risk for distant spread requiring systemic approaches to improve the likelihood of cure. Cystectomy is the standard approach for the treatment of invasive bladder cancer. As a single modality, however, results were disappointing, with high local failure rates and poor survival $(5,6)$.

Radiotherapy (RT) can be an effective alternative in medically inoperable patients, in poor performance status, who cannot tolerate a cystectomy or chemotherapy (7).

We completed a prospective study of localized bladder cancer elderly patients (cT1-2 No) at our institution. All patients received dose-escalated radiation using a hypofractionated RT schedule consisted of 36 Gy in 6 weekly fractions. The primary endpoints of this study were the assessment of symptom palliation and acute gastrointestinal (GI) and genitourinary $(\mathrm{GU})$ toxicity from patients treated only with 3D conformal Radiotherapy technique without previous cystectomy. The second endpoint was the evaluation of progression free survival (PFS).

\section{MATERIALS AND METHODS}

\section{Patient characteristics}

Clinical data of the 58 patients treated at University Hospital of Athens Attikon between February 2005 and June 2011 were collected. The pretreatment evaluation included medical history, physical examination, laboratory tests (including complete blood count, liver function tests, BUN, creatinine, alkaline phosphatase, uric acid), cystoscopy with bladder mapping, urine cytology and staging exams as computed tomography (CT) and/ or Magnetic Resonance (MRI) of the abdomen and pelvis. The patients' characteristics are shown in Table-1. The study was approved by the local ethical committee.

\section{Inclusion - exclusion criteria}

Eligible patients had histologically confurmed clinical localized Bladder Cancer Stage (cT12 N0) (according to American Joint Committee on Cancer staging manual, 7th edition, 2010). The patients included were medically inoperable with age more than 75 years and Karnofsky performance status lower than $70 \%$.

Patients were ineligible if they had undergone previous pelvic RT or cystectomy, lymph node metastatic involvement, distant metastases, or had a hip prosthesis.

\section{Treatment planning and Simulation}

Each patient underwent a virtual CT-simulation, in supine position, using "knee sponge" to consistently align lower extremities. Patients were instructed to have an empty bladder and rectum (following an enema) during simulation. Moreover, during the whole course of treatment, they were instructed to have certain dietary course with food including minimum fibers, in order to prevent diarrhea.

For treatment planning, a CT scan covering a region from the first lumbar vertebra to the lower part of the perineum was obtained for each patient. A conventional virtual CT simulation before computed tomography (CT) scan was performed to define preliminary isocenter and beam wi$\mathrm{dth}$. Patients were placed in a supine position, and a small amount of oral contrast was administered for the delineation of small bowel.

Planning CT scan of the pelvis was then performed with a $0.5 \mathrm{~cm}$ spacing between slices. The CT datasets were transferred to the Prosoma ${ }^{\circledR}$ virtual simulation system and after the delineation of the targets (clinical target volume - CTV, planning target volume - PTV) and the organs at risk (OAR), the images were transferred to Eclipse Varian Treatment Planning (Release 6.5, Varian Associates, Palo Alto, CA). Organ at risk (OAR) was defined to be an uninvolved organ that, if given 
Table 1 - Patients' characteristics.

\begin{tabular}{lc}
\hline Age median (range) & $77(70-91)$ \\
\hline T1 & 12 \\
T2 & 46 \\
PS & 10 \\
$\quad 60 \%-70 \%$ & 48 \\
$50 \%-60 \%$ & $47 / 11$ \\
Male / female
\end{tabular}

an excess radiation dose, might be damaged and would compromise the success of the course of radiation therapy.

The clinical target volume (CTV) was the bladder; the planning target volume (PTV) was obtained by expanding CTV with a margin of 1 $\mathrm{cm}$ in each direction, and of $0.5 \mathrm{~cm}$ posteriorly. In order to keep the bladder emptiness during treatment, the patients undergone their irradiation as soon as they have urinated. Rectum was manually contoured from the distal ischiatic branch to the sigmoid flexure. In addition small bowel and femoral heads were contoured. The entire bladder was treated, using a four field technique with 15 MV X-ray energy beams. The dose was prescribed according to ICRU criteria (8).

The dose constraints for the OARs are described below:

1. Rectum: V70 < 20-25\%, V50 < 50\%.

2. Femoral heads: V50 < 5\%.

3. Small bowel: V52 $=0 \%$.

Where Vx is the volume or the organ/target that receives the $\mathrm{x}$ dose.

In order to extrapolate the previous data according to our irradiation schedule, we used linear-quadratic (LQ) modeling in order to equate the hypofractionation schedules to the Normalised Total Dose (NTD) if delivered in 2Gy-fractions (9). Thus, NTD represents the dose given in 2Gy fractions that would give the equivalent biologic effect to the new hypofractionated dose:

$$
N T D=D_{\text {new }} \frac{d_{\text {new }}+\alpha / \beta}{2+\alpha / \beta}
$$

Where Dnew and dnew are respectively the total dose and dose per fraction for a suggested hypofractionation scheme. Normalized Total Dose - NTD has been calculated and tabulated for both bladder carcinona $(\alpha / \beta=10 \mathrm{~Gy})$ and normal tissues $(\alpha / \beta=3 \mathrm{~Gy})(10)$. Taking into account that $\alpha / \beta=10$, then NTD was 48Gy, while considering that $\alpha / \beta=3$, then NTD was $64.8 \mathrm{~Gy}$.

Patient setup was monitored weekly using portal films.

Before (at base line), during and 3 months after the completion of the treatment, the patients were evaluated by a radiation oncologist in terms of toxicity and symptom palliation. We used the RTOG/EORTC acute radiation morbidity scoring system for gastrointestinal (GI) and genitourinary (GU) toxicity grading (11). For the symptom palliation we used the incidence of haematuria, dysuria and frequency, while for the pain we used the visual analogue score (VAS) (12). In terms of heamaturia, dysuria and frequency, the patients were asked if there was a definite improvement in the above parameters or not.

\section{Statistical methods}

For the assessment of the statistical difference between VAS score before and after radiotherapy we used the Wilcoxon non-parametric test. For the correlation between symptom palliation and VAS score improvement, we used the spearman-rho test. The assessment of significant differences in symptom palliation before and after RT was performed with the chiv ${ }^{\mathrm{vv}}$ test. The level of statistical significance was set at the 0.05. All the statistical analysis was performed with the SPSS v.10 software (IL, USA).

\section{RESULTS}

The total number of patients with GI acute toxicity were 13 out 58 (22.4\%) and 5 out of 58 (5.6\%), for grade I and II respectively. The total number of patients with GU acute toxicity were 
19 out of 58 (32.7\%) and 10 out of 58 (17.2\%), for grade I and II respectively. No patients had Grade 3 or higher GI or GU toxicity scores. The detailed radiation induced toxicities are shown in Table-2. The treatment that was administered for the radiation toxicity was: non-steroids antinflammatory for dysuria, urgency, frequency, nocturia; diet, antidiarrheals for diarrhea; rectal non-steroids antinflammatory and corticosteroids for rectal irritation and pain.

In terms of clinical outcome, 55 out of 58 patients (94.8\%) reported palliation of haematuria, while 19 out of 58 reported no change in frequency and dysuria (Table-3). As shown in Figure-1, the VAS score significantly improved from $4.2( \pm 1.1)$ to $1.8( \pm 0.6)$, before and after RT. No correlation was noted between the VAS score and the other parameters palliation such as dysuria, frequency and haematuria. The median progression free survival (PFS) was 14 months.

\section{DISCUSSION}

In an attempt to improve external beam radiotherapy in bladder cancer, the current study investigated the possibility of shortening the days of irradiation. Shortening of the overall treatment time could increase the efficacy of the treatment but could also increase toxicity. The conventional fractionation of $2 \mathrm{~Gy} /$ day up to a total dose of $66 \mathrm{~Gy}$ seems quite equivalent in terms of radiobiology with the scheme of six fractions of 6Gy, according to linear-quadratic model, when the $\alpha / \beta$ ratio is taken equal to 3 (13). Comparing the conventional fractionation treatment scheme with the other hypofractionated schedules, since the blad$\operatorname{der} \alpha / \beta$ ratio is $10 \mathrm{~Gy}$, it comes out that short overall treatment times and large fraction sizes have no potential of increasing therapeutic ratio and therefore cannot be the treatment of choice.

However, there are pragmatic benefits in reducing the number of treatment sessions and travelling requirements for elderly patients and enabling better use of limited resources. We have to keep in mind that Greece is a Country with a lot of isolated villages either in the mountains or in the islands. Patients are often unable to undergone a schedule with 33 daily session for more than a month. Given also the often limited department resources and the long waiting lists, a reduction in the workload of the linear accelerator is often realized by hypofractionation. Moreover, one must remember that almost all studies that have investigated changes in the fractionation schedule were published before the widespread introduction of conformal radiotherapy. It is quite possible that

Table 2 - EORTC/RTOG acute radiation induced toxicity.

\begin{tabular}{lccc}
\hline & Grade 0 & Grade I & Grade II \\
\hline Gl acute toxicity & $40 / 58(68.9 \%)$ & $13 / 58(22.4 \%)$ & $5 / 58(5.6 \%)$ \\
GU acute toxicity & $29 / 58(50 \%)$ & $19 / 58(32.7 \%)$ & $10 / 58(17.2 \%)$ \\
\hline
\end{tabular}

Table 3 - Symptom palliation before and post RT.

\begin{tabular}{lccc}
\hline & Before RT & After RT & P (chi test) \\
\hline Heamaturia & $50 / 58(86.2 \%)$ & $3 / 58(5.2 \%)$ & $<0.01$ \\
Dysuria & $34 / 58(58.6 \%)$ & $19 / 58(32.7 \%)$ & \\
Frequency & $44 / 58(75.8 \%)$ & $19 / 58(32.7 \%)$ & \\
\hline
\end{tabular}


Figure 1 - Bladder related pain VAS scores before and after radiotherapy $(P<0.001$, Wilcoxon test).

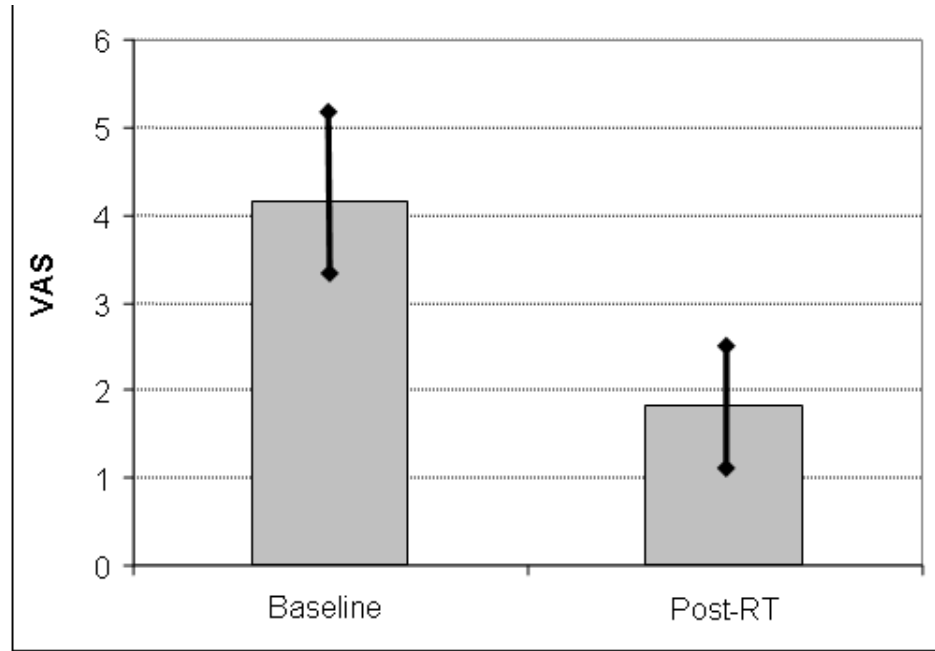

any improvement in therapeutic ratio resulting from optimizing the radiobiological parameters with altered fractionation may have been nullified by poor dose distributions, exceeding the normal tissue tolerance doses.

In our study the acute toxicity was mild, without any grade III morbidity noted, while the response to treatment was low with a median PFS of 15 months. There are several equivalent reports in the literature. A large study applying 36 Gy in six fractions in 18 days reported an acceptable severe late toxicity but only a 31\% 5 year local control rate (14). Pos et al. (15) treated 50 patients with a T2-T4 NOM0 transitional cell carcinoma of the bladder with a dose of 40 Gy in 2-Gy fractions. The irradiation was administered to the small pelvis with a concomitant boost limited to the bladder tumor area plus margin of 15 Gy in fractions of 0.75 Gy. The total tumor dose was 55 Gy in 20 fractions in 4 weeks. Severe acute toxicity G3 was observed in seven patients (14\%). Severe late toxicity G3 was observed in six patients (13\%). Thirty-seven patients (74\%) showed a complete and five $(10 \%)$ a partial remission after treatment.

In another trial, McLaren et al. (16) reported a study with 55 patients who underwent the same weekly scheme as ours with 6 Gy per session, while the total dose ranged from 60-66 Gy. Totally $64 \%$ of the patient noticed an improvement at a
1 month review. In the same way as in our study, $92 \%$ of patients with haematuria were completely palliated compared to only $24 \%$ of those with dysuria and frequency. Median overall survival was as low as 9 months (range 2-41months). However, the toxicity was higher than our reported rate, with a grade 3 acute urinary and bowel treatment related toxicity recorded in 18\% and 9\% of patients, respectively. However, no significant late toxicity (> grade 2) was recorded.

Patients offered defınitive irradiation have typically been nonsurgical candidates with various comorbidities and more advanced disease, contributing to poorer prognoses than those selected for surgery $(17,18)$. The 5-year survival rate for muscle-invasive bladder cancer treated with definitive external-beam radiotherapy alone is $20 \%$ to $40 \%$ (18). Similar to surgical series, survival rates following definitive radiotherapy have been higher in patients with invasion limited to the bladder compared with those with extravesical (T3b or T4) disease (16-20).

At last but not least we have to emphasize the possibility of hypofractionated radiotherapy with minimum sessions in a group of patients with poor prognosis (16) and with the inability to follow more than 5 weeks of daily irradiation schedules. In our study the patients had a mild toxicity with good palliation of symptoms. 


\section{CONCLUSIONS}

Our study showed that 3D-CRT is a feasible and safe modality allowing for hypofractionation up to 36 Gy .This study demonstrates that it is possible to deliver hypofractionated 3DCRT to the bladder with an acceptable acute toxicity rate in a poor prognostic patient group.

\section{ABBREVIATIONS}

RT: radiotherapy

GI: gastrointestinal

GU: genitourinary

CTV: clinical target volume

PTV: planning target volume

OAR: organs at risk

CT: computerized tomography

VAS: visual analogue score

PFS: progression free survival.

\section{CONFLICT OF INTEREST}

\section{None declared.}

\section{REFERENCES}

1. Jemal A, Siegel R, Xu J, Ward E: Cancer statistics, 2010. CA Cancer J Clin. 2010; 60: 277-300. Erratum in: CA Cancer J Clin. 2011; 61: 133-4.

2. Siegel $R$, Naishadham D, Jemal A: Cancer statistics, 2012. CA Cancer J Clin. 2012; 62: 10-29.

3. Howe HL, Wu X, Ries LA, Cokkinides V, Ahmed F, Jemal A, et al.: Annual report to the nation on the status of cancer, 19752003, featuring cancer among U.S. Hispanic/Latino populations. Cancer. 2006; 107: 1711-42.

4. Silverman DT, Hartge P, Morrison AS, Devesa SS: Epidemiology of bladder cancer. Hematol Oncol Clin North Am. 1992; 6: 1-30.

5. Gospodarowicz MK, Hawkins NV, Rawlings GA, Connolly JG, Jewett MA, Thomas GM, et al.: Radical radiotherapy for muscle invasive transitional cell carcinoma of the bladder: failure analysis. J Urol. 1989; 142: 1448-53; discussion 1453-4.

6. Duncan W, Quilty PM: The results of a series of 963 patients with transitional cell carcinoma of the urinary bladder primarily treated by radical megavoltage X-ray therapy. Radiother Oncol. 1986; 7: 299-310.

7. Rödel C, Grabenbauer GG, Kühn R, Papadopoulos T, Dunst $\mathrm{J}$, Meyer $\mathrm{M}$, et al.: Combined-modality treatment and selective organ preservation in invasive bladder cancer: long-term results. J Clin Oncol. 2002; 20: 3061-71.
8. International Commission on Radiation Units and Measurements. Report 50: Prescribing, recording and reporting photon beam therapy. Bethesda: ICRU, 1993.

9. Fowler JF: The linear-quadratic formula and progress in fractionated radiotherapy. Br J Radiol. 1989; 62: 679-94.

10. Emami B, Lyman J, Brown A, Coia L, Goitein M, Munzenrider JE, et al:: Tolerance of normal tissue to therapeutic irradiation. Int J Radiat Oncol Biol Phys. 1991; 21: 109-22.

11. Cox JD, Stetz J, Pajak TF: Toxicity criteria of the Radiation Therapy Oncology Group (RTOG) and the European Organization for Research and Treatment of Cancer (EORTC) Int J Radiat Oncol Biol Phys. 1995; 31: 1341-6.

12. Lasagna L: The clinical measurement of pain. Ann N Y Acad Sci. 1960; 86: 28-37.

13. Jones B, Dale RG, Deehan C, Hopkins KI, Morgan DA: The role of biologically effective dose (BED) in clinical oncology. Clin Oncol (R Coll Radiol). 2001; 13: 71-81.

14. Scholten AN, Leer JW, Collins CD, Wondergem J, Hermans J, Timothy A: Hypofractionated radiotherapy for invasive bladder cancer. Radiother Oncol. 1997; 43: 163-9.

15. Pos FJ, van Tienhoven G, Hulshof MC, Koedooder K, González González D: Concomitant boost radiotherapy for muscle invasive bladder cancer. Radiother Oncol. 2003; 68: 75-80.

16. McLaren DB, Morrey D, Mason MD: Hypofractionated radiotherapy for muscle invasive bladder cancer in the elderly. Radiother Oncol. 1997; 43: 171-4.

17. Munro NP, Sundaram SK, Weston PM, Fairley L, Harrison SC, Forman $D$, et al:: A 10-year retrospective review of a nonrandomized cohort of 458 patients undergoing radical radiotherapy or cystectomy in Yorkshire, UK. Int J Radiat Oncol Biol Phys. 2010; 77: 119-24.

18. Marta GN, Hanna SA, Gadia R, Correa SF, Silva JL, de Andrade Carvalho $\mathrm{H}$ : The role of radiotherapy in urinary bladder cancer: current status. Int Braz J Urol. 2012; 38: 144-53; discussion 153-4.

19. Leliveld AM, Bastiaannet E, Doornweerd BH, Schaapveld M, de Jong IJ: High risk bladder cancer: current management and survival. Int Braz J Urol. 2011; 37: 203-10; discussion 210-2.

20. Nakamura RA, Monti CR, Castilho LN, Trevisan FA, Valim $A C$, Reinato JÁ: Prognostic factors for late urinary toxicity grade 2-3 after conformal radiation therapy on patients with prostate cancer. Int Braz J Urol. 2007; 33: 652-9; discussion 660-1. 